\title{
Dosimetric analysis and clinical outcomes in CT-based mould brachytherapy in early oral cancers in patients unfit for surgery
}

\author{
Ashutosh Mukherji, MD!, Sinnatamby Mourougan², Kandasamy Saravannan², Singhavajala Vivekanandam! \\ K. Sathyanarayana Reddy ${ }^{1,3}$ \\ 'Department of Radiotherapy, Regional Cancer Centre, JIPMER, Puducherry, ${ }^{2}$ Medical Physics, Regional Cancer Centre, JIPMER, Puducherry, \\ ${ }^{3}$ Oncology, Mahatma Gandhi Institute, Puducherry, India
}

\begin{abstract}
Purpose: Brachytherapy in the oral cavity is an important alternative to conventional treatment, and provides a high localized dose and short overall treatment time. A rapid fall of dose beyond radioactive source makes it possible for increased tumour control and sparing surrounding tissue, while short overall treatment duration reduces risk of tumour repopulation. Moulds are fabricated to hold the catheters in position as closely as possible to tumour surface to provide adequate dose coverage of tumour volume and increase distance to other normal surrounding structures. Image based planning and dose optimisation help in better defining target volume and dose coverage.

Material and methods: A retrospective analysis of patients of early squamous cell carcinomas of lip and buccal mucosa from September 2011 to June 2014 to study response to mould brachytherapy. Double plane moulds were prepared for all lip cancer cases and single plane for buccal mucosa cases. Patients are being followed up till disease recurrence. In this study evaluation was done of the technique used, planning details, response to therapy, and reactions encountered.

Results: Nine patients treated by mould therapy were reviewed; seven cases were of lip and two of buccal mucosal cancers. Dose delivered ranged from 12.5-48 Gy in fraction sizes of 2.5-3.5 Gy. Equivalent dose in $2 \mathrm{~Gy}$ fractions (EQD2) ranged from 18-64 Gy. Maximum dose to organs at risk (OAR) was 91\% of prescribed dose. Local mucositis was only reaction in all cases, which resolved in 3-6 weeks. Median follow-up was 19 months. Eight out of nine patients are in remission at a minimum of 7 months (1 case, rest over 14 months) post therapy and only one patient had nodal recurrence at 18 months.

Conclusions: Mould therapy is an effective treatment method for selected early and superficial squamous cell carcinomas of the oral cavity, although indications are limited.
\end{abstract}

Key words: brachytherapy, buccal cancer, lip cancer, mould brachytherapy, oral cancer.

\section{Purpose}

Brachytherapy in head and neck cancers especially in the oral cavity is an important alternative to conventional external beam radiotherapy. It provides a high localized dose of radiation, with rapid fall-off and short overall treatment time [1]. Brachytherapy can be applied as a sole treatment, instead of surgery, or as local boost in combination with external beam radiation therapy (EBRT). Mould brachytherapy is a technique of delivering brachytherapy by an applicator called mould that is usually custom made and designed to provide a more constant and reproducible frame for source positioning [2]. Oral cancers account for $5-7 \%$ of all cancers. With brachytherapy, sur- gery is avoided by that preserving the normal structure and function of the oro-masticatory complex [3]. A rapid fall in dose beyond radioactive source makes it possible for increased tumour control and sparing the surrounding tissue, while shorter overall treatment duration reduces risk of tumour cell repopulation [2]. During this short span it is important to ensure that the catheters remain in the exact position determined by the plan prescribed. Hence, these customized devices or moulds are designed to hold the catheters in position as closely as possible to tumour surface to provide adequate dose coverage of tumour volume and increase the distance to other normal surrounding structures. Local control rate is more than $90 \%$ for T1, and T2N0 tumours treated with brachyther- 
apy alone [1]. The control rate is lower in larger tumours treated with EBRT and brachytherapy boost.

Mould brachytherapy alone in early stage head and neck cancers has so far limited indications. Mould therapy after chemo-radiation is indicated in previously untreated superficial squamous cell carcinomas of the lip, floor of mouth, soft palate or gingiva, $\mathrm{T} 1 / 2$ tumours, and tumours showing complete response at the end of chemo-radiotherapy. In this study, we have tried to analyse the effectiveness of mould brachytherapy offered in our institute to patients with early stage of oral cancers who could not otherwise undergo surgery, especially with the use of a thermoplastic frame for making the mould.

\section{Material and methods}

\section{Patient profile}

A retrospective analysis was done for patients with early stage squamous cell carcinomas of the lip and buccal mucosa from September 2011 to June 2014 to study the response to curative mould brachytherapy. All selected patients at the time of presentation first underwent a complete local and regional examination of the oral cavity, oropharynx, larynx, and hypopharynx including endoscopic assessment and imaging by contrast-enhanced computed tomography to confirm the clinical diagnosis of early stage disease or small localised recurrence. All but one of these patients had not been found fit for general anaesthesia for surgery or interstitial brachytherapy, and were considered for mould brachytherapy. Only one patient had been considered fit for surgery and had undergone surgical excision. In all cases, the thickness of the lesion was less than $0.5 \mathrm{~cm}$. Treatment technique and expected results as well as complications were explained in all cases and consent taken. After dental review and any required extractions, patients were taken up for mould preparation.

Nine patients treated by mould therapy during this period were reviewed; seven patients with lip and two with buccal mucosal cancers. Of the seven lip cancer patients, five patients had stage 1 disease, and two patients had stage 2 disease. Both patients with buccal mucosal cancers were recognised as recurrent diseases having already undergone surgery and received adjuvant radiotherapy. Of the seven lip cancer patients, three patients received mould therapy as an initial boost followed by external beam radiotherapy. One lip cancer patient received mould brachytherapy as adjuvant radiotherapy following wide excision for a T1N0 lip cancer with deep resected margin involved by tumour, while the remaining three received mould brachytherapy as a single modality treatment. Six of the studied cases were males, while three were females. All cases except two were above 65 years of age at presentation with the mean age being 62 years $(S D \pm 10.98)$ (Table 1$)$. The earliest case was treated in September 2011, and the last one completed treatment in June 2014. Eight of the cases during work-up could not undergo surgery because of co-existing morbidities (bronchial asthma in one case, cardiac ailments in four others) or because of advanced age and poor general condition. The ninth case presented post-surgery with involved deep margin.

\section{Mould preparation}

The moulds were prepared from a framework of thermoplastic sheet moulded to patient anatomy, and then covered by thin layer of dental wax and customised for each patient. The thickness of each plane of the wax mould was between 6-7 $\mathrm{mm}$ for all cases. Double plane moulds, with one plane on the skin surface and one plane

Table 1. Patient description and dose parameters

\begin{tabular}{|c|c|c|c|c|c|c|c|c|c|}
\hline \multirow[t]{2}{*}{ Age } & \multirow[t]{2}{*}{ Sex } & \multirow{2}{*}{$\begin{array}{l}\text { Site of } \\
\text { lesion }\end{array}$} & \multirow[t]{2}{*}{ Stage } & \multirow{2}{*}{$\begin{array}{l}\text { Previous } \\
\text { treatment } \\
\text { received }\end{array}$} & \multicolumn{2}{|c|}{ Brachytherapy dose } & \multirow{2}{*}{$\begin{array}{l}\text { EBRT - total } \\
\text { dose and } \\
\text { fractions }\end{array}$} & \multirow{2}{*}{$\begin{array}{l}\text { Prescribed } \\
\text { isodose to } \\
\text { PTV (\%) }\end{array}$} & \multirow[t]{2}{*}{ EQD2 (Gy) } \\
\hline & & & & & $\begin{array}{c}\text { Total dose } \\
\text { (dose/\#) (Gy) }\end{array}$ & Fractions & & & \\
\hline 70 & M & lip & cT1NO & none & $21(3)$ & 7 & $\begin{array}{l}40 \text { Gy/20 } \\
\text { fractions }\end{array}$ & 60 & $29.75+40$ \\
\hline 45 & $\mathrm{~F}$ & lip & cT2NO & none & $45(3)$ & 15 & NA & 75 & 63.75 \\
\hline 75 & M & $\begin{array}{l}\text { buccal } \\
\text { mucosa }\end{array}$ & recurrence & RT & $38.5(3.5)$ & 11 & NA & 80 & 54.54 \\
\hline 65 & M & $\begin{array}{l}\text { buccal } \\
\text { mucosa }\end{array}$ & recurrence & surgery + RT & $37.5(2.5)$ & 15 & NA & 85 & 53.12 \\
\hline 65 & $\mathrm{~F}$ & lip & cT1NO & none & $45.5(3.5)$ & 13 & NA & 80 & 54.54 \\
\hline 68 & M & lip & cT1NO & none & $12.5(2.5)$ & 5 & $\begin{array}{l}46 \text { Gy/23 } \\
\text { fractions }\end{array}$ & 80 & $17.71+46$ \\
\hline 66 & $\mathrm{~F}$ & lip & cT1NO & none & $45(2.5)$ & 18 & NA & 80 & 63.75 \\
\hline 65 & M & lip & CT2NO & none & $17.5(2.5)$ & 7 & $\begin{array}{l}44 \mathrm{~Gy} / 22 \\
\text { fractions }\end{array}$ & 85 & $24.79+44$ \\
\hline 40 & $M$ & lip & pT1NO & $\begin{array}{l}\text { surgery } \\
(\mathrm{DRM}+)\end{array}$ & $48(3)$ & 16 & NA & 80 & 56 \\
\hline
\end{tabular}

$M$ - male, F-female, RT - radiotherapy, EBRT - external beam radiation therapy, PTV - planning target volume, EQD2 - equivalent (isoeffective) dose in 2 Gy fractions 
along mucosal surface, were used in seven cases that were lip cancers and where the tumour tissue could be sandwiched between the two mould planes. Two cases of recurrent buccal mucosal cancers with localized disease were treated with mould brachytherapy with the mould placed as a single plane application directly opposed to the lesion. After examining and marking the lesion by the oncologist, the physicist made an initial cut-out for the mould with thermoplastic sheet. Then wax was heated in a water heater and cast in the required shape over the thermoplastic according to local patient anatomy. The shaped wax mould was then approximated to the lesion in patient treatment position with immobilisation devices in situ. The prepared mould was matched with lesion surface and local anatomy; edges were trimmed and smoothened. Required positions as well as the number of catheters were marked into the mould. Catheters were then fixed to the mould, which was then again positioned on the patient to check feasibility of catheter placement and lesion coverage. The lesion was then marked with lead wires and CT-simulation of patient in the treatment position done (Fig. 1A-F).

\section{Treatment planning and delivery}

After CT-simulation, image reconstruction, target volume contouring, and treatment planning was done with Eclipse TPS version 10.0 (Varian Medical Systems, Inc., USA). Treatment delivery was done with GammaMedplus iX HDR after loader (Varian Medical Systems, Inc., USA) with Ir-192 stepping source. The target volume was defined as the volume encompassing the tumour/ tumour bed with a margin of approximately $1-1.5 \mathrm{~cm}$, specified by the oncologist. The dosimetry was calculated using volume optimization techniques. Dose was prescribed to $80-85 \%$ isodose line in most cases in order to cover maximum tumour volume. It was also our aim to keep the volume of tissue receiving dose greater than $200 \%$ of the prescribed dose to less than $5 \%$ and further higher doses to within substance of the mould (Table 2, Fig. 2A-C). Treatments were delivered twice daily with six hour's break. Analysis of reactions was done every third day and at the end of the treatment. After that, patients were reviewed monthly for first six months and then every three months till disease recurrence. Evaluation was done on the base of planning details, response to therapy and reactions encountered.

\section{Dosimetric analysis}

Dose delivered ranged from 12.5-48 Gy in fraction sizes of 2.5-3.5 Gy given twice daily. Three patients who received mould therapy as boost then received EBRT for further four to four and half weeks; two weeks after completion of mould therapy. These three patients completed their mould treatment in two-three days. Brachytherapy part EQD2 (equivalent dose in 2 Gy fractions) of these three patients were 17.71 Gy, 24.75 Gy and 29.75 Gy. The remaining six patients who received mould therapy as the definitive treatment completed their schedules in 1118 fractions over six to nine days. EQD2 of these patients ranged from 54 Gy for recurrent disease cases to 64 Gy for radically treated cases (Table 1 ).

Biological effective doses (BEDs) (for 3 and $10 \mathrm{~Gy}$ ) were also calculated in all cases, and the mean BED doses were 66.78 Gy (SD \pm 27.505$)$ for BED $3 \mathrm{~Gy}$ and $43.66 \mathrm{~Gy}$ (SD \pm 16.358 ) for BED $10 \mathrm{~Gy}$. This was less than $100 \mathrm{~Gy}$ in all cases indicating less risk for late sequelae (Table 2). The volume of PTV covered by the prescribed isodose, volume receiving $90 \%, 150 \%$ and $200 \%$ of prescribed dose were also assessed with mean ( \pm SD) being $89 \%( \pm 7.7 \%)$, $85 \%( \pm 9.3 \%), 11 \%( \pm 8 \%)$ and $2 \%( \pm 1.8 \%)$ respectively (Table 2). The average maximal tissue-mould interface dose was $148 \%$ (SD $\pm 24 \%$ ). In all cases, the organ at risk was the mandible, and it was contoured to determine the dose received. Maximum dose to OAR (organs at risk) was $91 \%$ of the prescribed dose to 2 cc of OAR with the median dose being 64\% (range 4-91\%) (Table 2).

\section{Results}

\section{Response to therapy}

All patients had immediate complete response that was maintained over the follow-up period in eight out of the nine treated cases. Only one patient with an initial lip lesion developed a recurrence in the ipsilateral submandibular node at 18 months after therapy that was treated by surgery. The patient is alive and still under follow-up with no further nodal or local recurrence. The other patients have maintained their remission. All patients are still under follow-up with the median duration of follow-up being 19 months (range 7-34 months) (Fig. 3). Only one patient has completed less than 12 months follow-up (at 7 months), six cases between 13-30 months, and two cases have completed more than 30 months follow-up.

\section{Treatment related sequelae}

Local lip surface mucosal inflammation (grade 1 in all nine cases and grade 2 in two cases) and surrounding skin reactions (grade 1 in all eight cases and grade 2 in three cases) were the treatment-related sequelae seen in all cases. These started occurring in all cases towards the end of therapy and in all cases reached their maximum severity at four week's post radiotherapy (Fig. 4). After that, the reactions resolved in the following 3-6 weeks. There were no late sequelae noted like ulcers, necrosis or strictures in any of the cases (eight of the nine cases have completed one-year treatment follow-up). However, superficial skin hypopigmentation was seen in four of the eight cases, which have completed at least one-year follow-up (Fig. 5).

\section{Discussion}

Radiotherapy is known to be highly effective in the treatment of superficial cancers of the head and neck [4]. Mould therapy is excellent in the treatment of superficial oral cancers as it provides a high localized dose of radiation, with rapid fall-off and short overall treatment time [1]. This technique enables highly reproducible ir- 

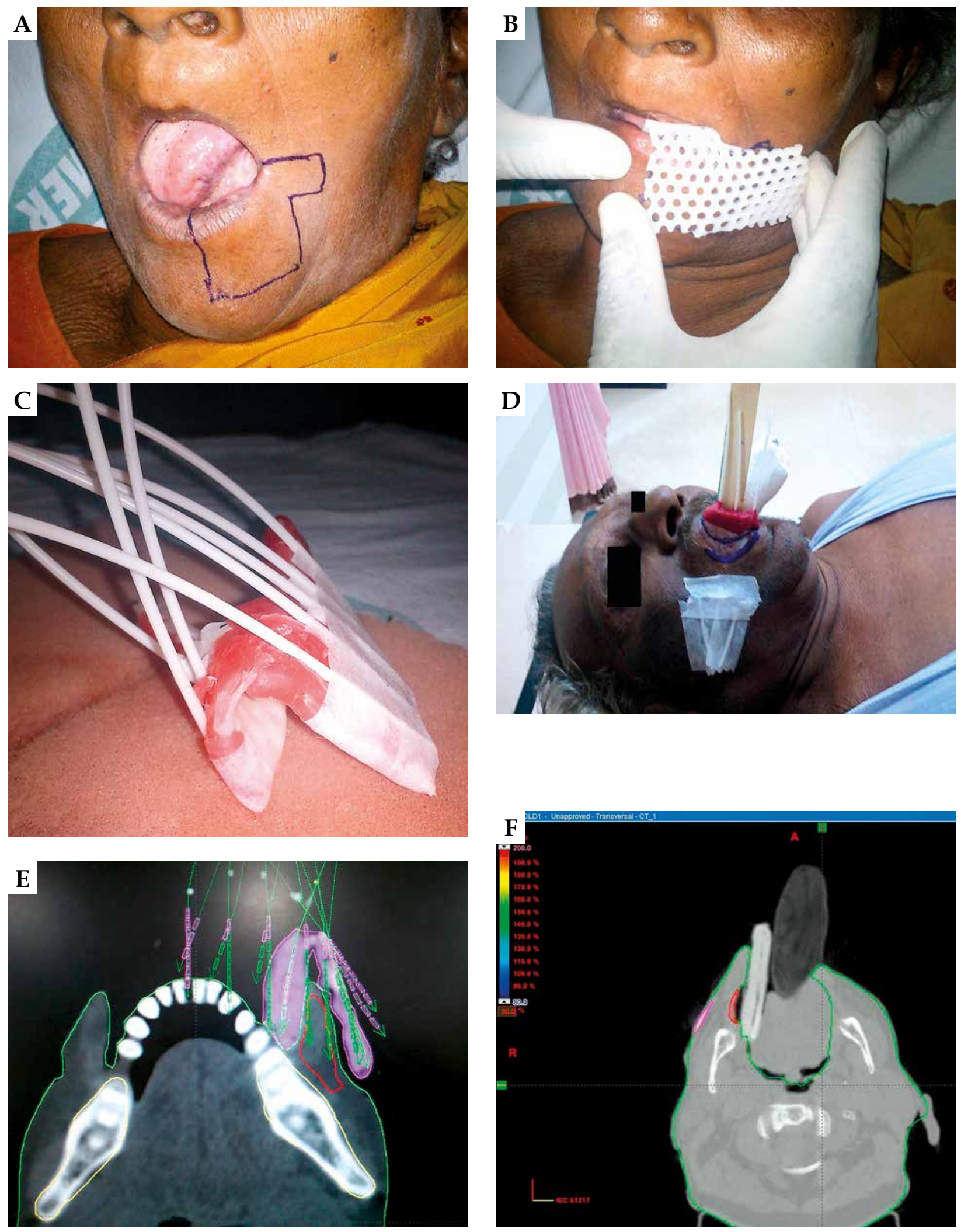

Fig. 1. A) Lesion marked by ink before mould preparation. B) Thermoplastic frame prepared and matched with local anatomy (with permission from corresponding author). C) Frame covered with dental wax and catheters fixed into the mould (with permission from corresponding author). D) Mould is approximated to lesion with lead wires on the skin surface to mark out the lesion before computed tomography (CT) scan and planning. E) Computed tomography simulation image with catheter reconstruction and planning target volume contoured. F) Single plane mould is approximated to lesion after CT scan and planning 
Table 2. Equivalent doses and dose volume distributions

\begin{tabular}{|c|c|c|c|c|c|c|c|c|c|}
\hline \multirow[t]{2}{*}{ Case } & \multirow{2}{*}{$\begin{array}{c}\text { Prescribed } \\
\text { isodose to } \\
\text { PTV (\%) }\end{array}$} & \multirow[t]{2}{*}{ BED 3 Gy } & \multirow[t]{2}{*}{ BED 10 Gy } & \multicolumn{4}{|c|}{ Dose in PTV } & \multirow{2}{*}{$\begin{array}{c}\mathrm{D}_{2 c c} \text { to } \mathrm{OAR} \\
\left(\mathrm{D}_{\max }\right)(\%)\end{array}$} & \multirow{2}{*}{$\begin{array}{c}\text { Max tissue } \\
\text { mould interface } \\
\text { dose (\%) }\end{array}$} \\
\hline & & & & $V_{200 \%}$ & $\mathrm{~V}_{150 \%}$ & $V_{90 \%}$ & $\mathrm{~V}_{\text {prescribed\% }}$ & & \\
\hline 1 & 60 & 42 & 27.30 & 0 & 9 & 80 & 83 & 48 & 150 \\
\hline 2 & 75 & 90 & 58.50 & 0 & 2 & 70 & 75 & 62 & 120 \\
\hline 3 & 80 & 83.42 & 52 & 2 & 10 & 90 & 90 & 70 & 150 \\
\hline 4 & 85 & 68.75 & 47 & $1-2$ & 25 & 90 & 90 & 91 & 150 \\
\hline 5 & 80 & 83.42 & 52 & 3 & 20 & 85 & 88 & 66 & 130 \\
\hline 6 & 80 & 22.90 & 15.63 & 5 & 20 & 90 & 98 & 89 & 190 \\
\hline 7 & 80 & 82.50 & 56.25 & 3 & 11 & 88 & 96 & 59 & 180 \\
\hline 8 & 80 & 96 & 62.40 & 0 & 1 & 70 & 80 & 28 & 130 \\
\hline 9 & 85 & 32 & 21.87 & 0 & 3 & 100 & 100 & 4 & 130 \\
\hline Mean & 78.3 & 66.777 & 43.661 & 2 & 11 & 85 & 89 & 57 & 148 \\
\hline SD & 7.1 & 25.705 & 16.358 & 1.8 & 8 & 9.3 & 7.7 & 26.2 & 22 \\
\hline
\end{tabular}

PTV - planning target volume, BED - biologically effective dose, OAR - organs at risk, $D_{\text {max }}$ - maximum dose, $V_{90}$ - volume of the anatomic volume receiving $90 \%$ of the prescribed dose, $V_{100}$-volume of the anatomic volume receiving $100 \%$ of the prescribed dose, $V_{150}$-volume of the anatomic volume receiving $150 \%$ of the prescribed dose

radiation $[5,6]$. Superficial lesions usually have a higher cure rate with radiation in comparison to deeply infiltrating lesions [4]. In our study also of the eight patients who have completed treatment and at least one-year follow-up, seven have remained disease free, while one patient had nodal recurrence at eighteen months.

But there is a paucity of both literature on the use of HDR (high-dose-rate) mould brachytherapy and the optimal time, dose, and fractionation guidelines $[7,8]$. The largest series in HDR brachytherapy so far has been by Guinot et al. [9] who studied the results of interstitial brachytherapy in 39 patients, and reported three-year cause-specific survival and local control of 91 and $88 \%$, respectively. However, there is no similar significant series in mould brachytherapy mainly because the indications for this technique are limited. The dose fraction sizes used by us in this review were based on our institute experience with interstitial brachytherapy as well as available literature.

A study by Nishimura et al. [10] took stock of toxicity and efficacy of HDR intra-cavitary brachytherapy using moulds in the treatment of squamous cell carcinoma of the oral cavity. Eight patients with squamous cell carcinoma of the oral cavity tumours, including the buccal mucosa, oral floor, and gingiva were treated. There was a good initial complete response rate in seven cases $(88 \%)$, but local recurrence was seen in four of these seven patients. Marginal recurrence occurred in two cases where retromolar trigone was involved. No severe late radiation damage was observed in the follow-up period of 15-57 months. This study concluded that while HDR brachytherapy with mould technique is a safe for early and superficial oral cavity cancers but it may not be sufficient for thick tumours or those located in the retromolar trigone. These findings have also been reproduced in our study with excellent initial results and with proper case selection results have been maintained over extended follow-up periods. Also, no significant late sequelae have been noted in our cohort of patients so far.

Obinata et al. presented their clinical experience with HDR brachytherapy for head and neck cancer with customized intraoral mould technique [11]. Two patients were treated with dental prostheses as the moulds for HDR brachytherapy and suggested it as a viable treatment option. Kudoh et al. introduced a mould treatment for maxillary gingival carcinoma [12]. Two patients with maxillary gingival carcinoma were given salvage treatment by this technique. The dose to the buccal mucosa and tongue were measured on the inner and outer surfaces of the mould before starting HDR brachytherapy, and was reduced to $10 \%$ of tumour dose. No recurrence or severe adverse effects to adjacent normal soft tissue were observed. It was concluded that HDR brachytherapy using customized intraoral mould designed by Kudoh et al. [12] might be a treatment option even in definitive therapy for maxillary gingival carcinoma. Chatani et al. [13] in a study of nine cases reported that mould therapy after chemo-radiotherapy is non-invasive and yields a reproducible distribution of the radiation dose tightly fitting tumour volume. Matsuzaki et al. [14] showed that HDR brachytherapy with customized mould is minimally invasive in oral cancers, but is difficult technically in buccal mucosal and lip cancers involving the commissural labiorum. This study concluded that HDR brachytherapy using customized moulds is a viable option for patients with buccal and lip cancers in those whom age and performance status limits the use of other therapeutic modalities, and other factors. Thus, we can see from the above examples that there is limited experience with mould brachytherapy in literature, and no universal recommendations have been put forward. Most patients in our study would have undergone surgical excision had 

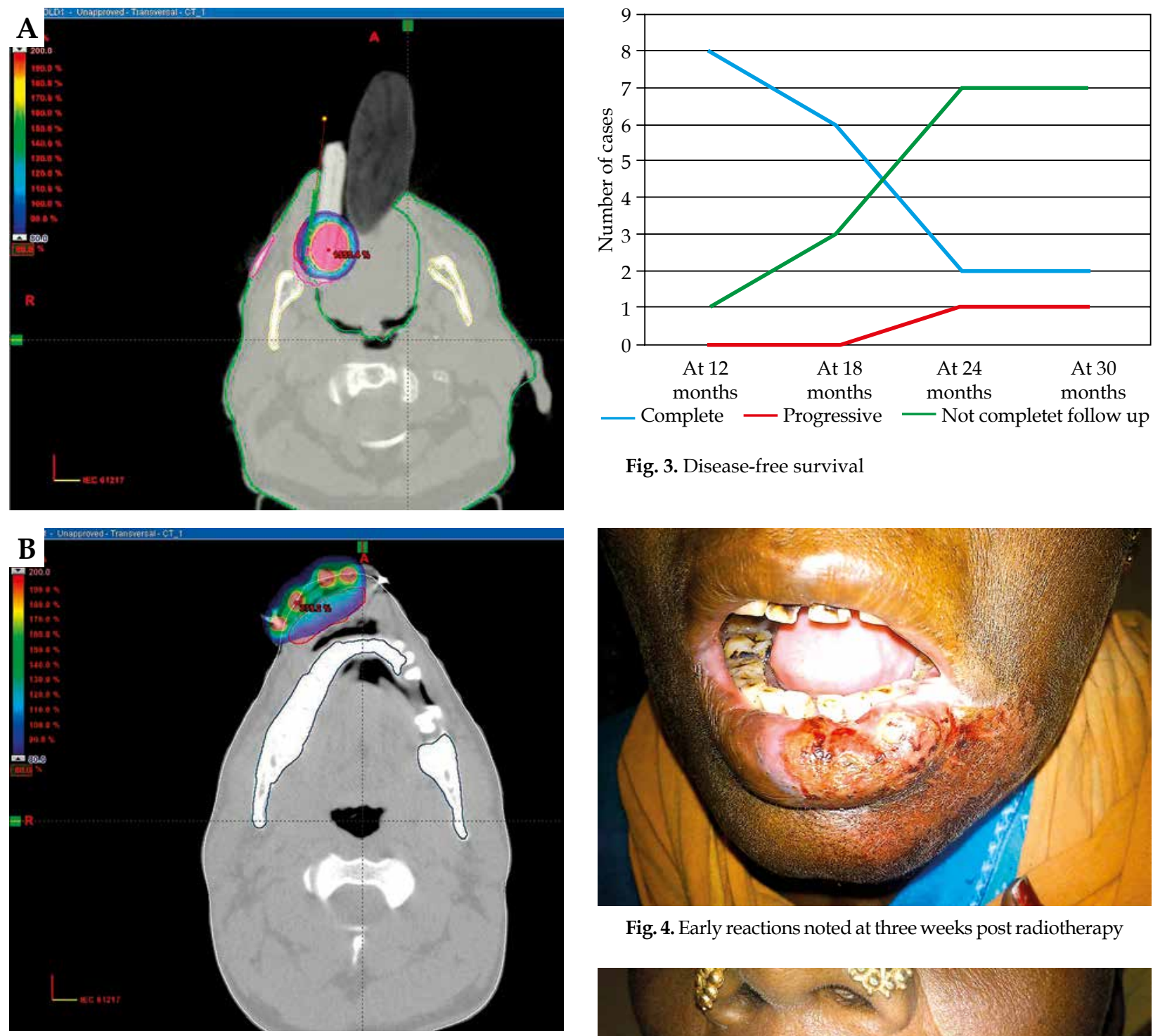

Fig. 3. Disease-free survival

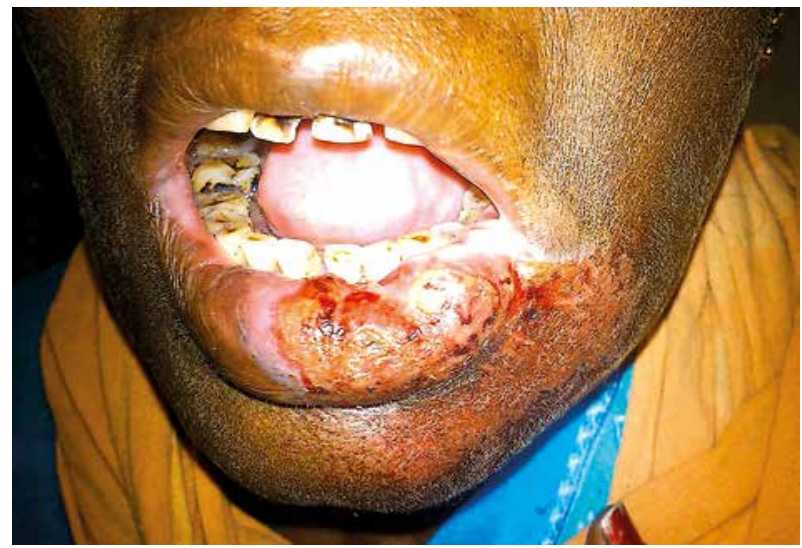

Fig. 4. Early reactions noted at three weeks post radiotherapy
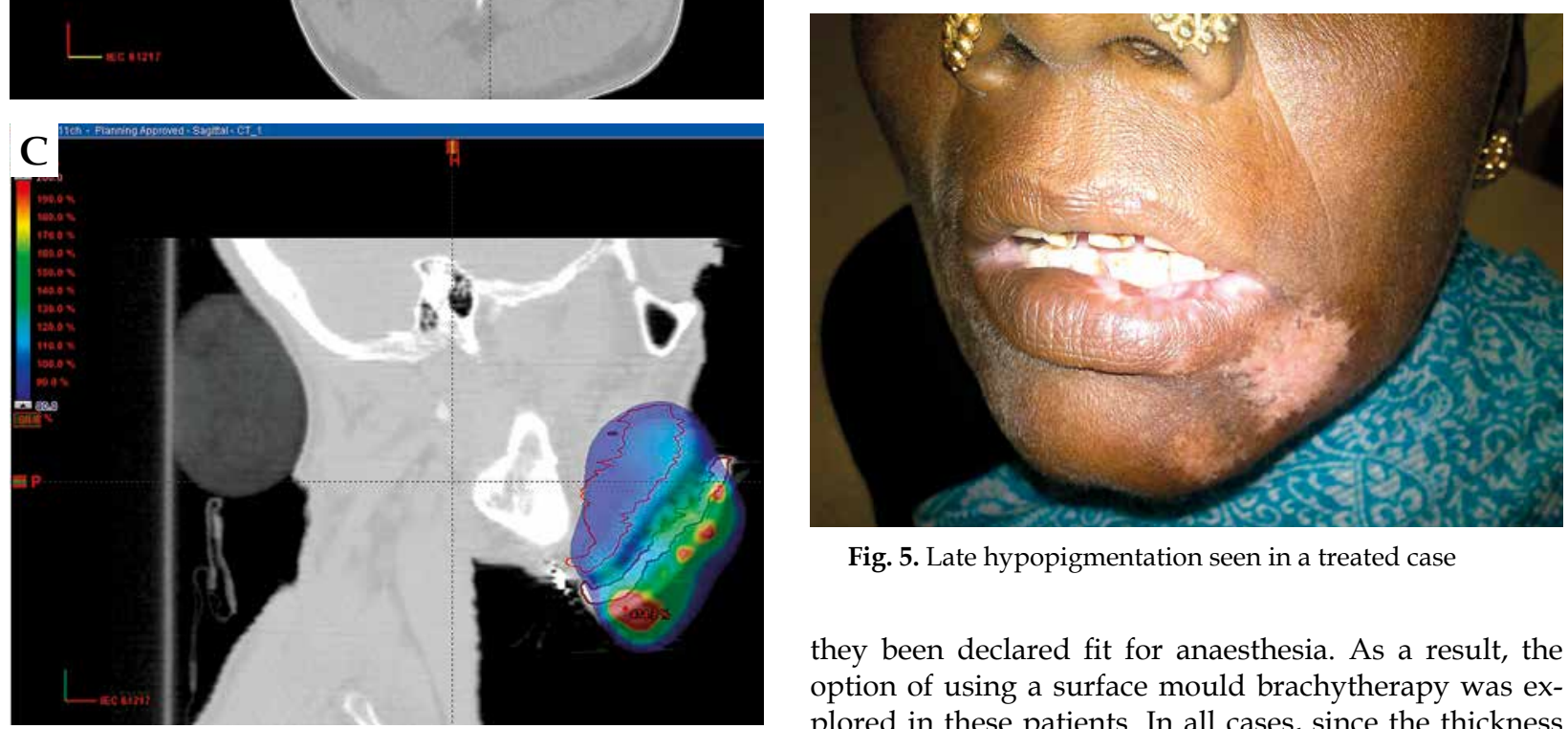

Fig. 5. Late hypopigmentation seen in a treated case

they been declared fit for anaesthesia. As a result, the option of using a surface mould brachytherapy was explored in these patients. In all cases, since the thickness of the lesions was less than $0.5 \mathrm{~cm}$, there was no problem in covering the clinical target volume (CTV) with the prescribed isodose. This has also been recommended in the GEC-ESTRO (Groupe Européen de Curiethérapie - European Society for Radiation Oncology) recommenerage by prescribed isodose. B) Dose wash showing lip lesion coverage by prescribed isodose. C) Dose colour-wash showing lip lesion coverage by prescribed isodose 
dations for prescribing mould brachytherapy [1]. All of the above-mentioned studies have used fraction sizes of 3-4 Gy delivered twice a day six hours apart to a total dose of 60 Gy low-dose-rate (LDR) equivalent in radical setting. We also used fraction sizes of 2.5-3.5 Gy delivered twice daily, six hours apart to an EQD2 dose of about $60 \mathrm{~Gy}$.

The advantages of mould brachytherapy include eliminating the morbidities from surgery, preserving function of oro-masticator space, outpatient treatment procedure, and simple, reproducible non-invasive treatments. In addition, in recent years advances in HDR brachytherapy such as the integration of imaging and optimization of dose distribution by improved planning systems have helped in better tumour localization and normal tissue definition. Additionally, it helps in improving dose distribution to the tumour and reducing normal tissue exposure [15]. In our study, also CT-based planning helped identify volumes of tissue receiving dose greater than $150 \%$ and optimize accordingly. We optimized our plans to try and keep areas receiving more than $200 \%$ within the mould. We also used a basic frame of thermoplastic over which layers of dental wax were added to obtain a mould 6-7 mm thick. Into these, the catheters were inserted to maintain a minimum distance of $0.5 \mathrm{~cm}$ from the skin, as per standard mould or intraluminal brachytherapy prescription guidelines [1].

\section{Conclusions}

Mould therapy can be an effective treatment method for selected early and superficial squamous cell carcinomas of the oral cavity, although indications are limited. It can be a cost effective method of treating radically or boosting early cancers of accessible regions in the oral cavity. Computed tomography-based planning in our study helped identify volumes of tissue receiving dose greater than $150 \%$ and optimizing accordingly. Thus, the risk of severe late sequelae following higher point doses can be avoided.

\section{Disclosure}

Authors report no conflict of interest.

\section{References}

1. Mazeron JJ, Ardiet JM, Haie-Méder C et al. GEC-ESTRO recommendations for brachytherapy for head and neck squamous cell carcinomas. Radiother Oncol 2009; 91: 150-156.

2. Ariji E, Hayashi N, Kimura $\mathrm{Y}$ et al. Customized mould brachytherapy for oral carcinomas through use of high-doserate remote afterloading apparatus. Oral Surg Oral Med Oral Pathol Oral Radiol Endod 1999; 87: 508-512.

3. Obinata $\mathrm{K}$, Ohmori $\mathrm{K}$, Tuchiya $\mathrm{K}$ et al. Clinical study of a spacer to help prevent osteoradionecrosis resulting from brachytherapy for tongue cancer. Oral Surg Oral Med Oral Pathol Oral Radiol Endod 2003; 95: 246-250.

4. Chalian VA, Drane JB, Standish SM. Maxillofacial prosthetics. William and Wilkins Company, Baltimore 1972; 181-183.

5. Rustgi SN, Cumberlin RL. An afterloading 192-Ir surface mould. Med Dosim 1993; 18: 39-42.

6. Takeda M, Shibuya H, Inoue T. The efficacy of gold-198 grain mould therapy for mucosal carcinomas of the oral cavity. Acta Oncol 1996; 35: 463-467.
7. Fietkau R. Brachytherapy for head and neck tumours. Activity Selectron Brachytherapy J 1993; 27: 69-72.

8. Otti M, Stuckischweiger G, Danninger R, et al. HDR brachytherapy for hard palate carcinoma. Activity Selectron Brachytherapy J 1992; Suppl 3: 26-28.

9. Guinot JL, Arribas L, Chust ML, et al. Lip cancer treatment with high dose rate brachytherapy. Radiother Oncol 2003; 69: 113-115.

10. Nishimura Y, Yoshihiko Yokoe $Y$ et al. High-dose-rate brachytherapy using moulds for oral cavity cancer: The technique and its limitations. Int J Clin Oncol 1998; 3: 351-356.

11. Obinata K, Ohmori K, Shirato $\mathrm{H}$ et al. Experience of highdose-rate brachytherapy for head and neck cancer treated by a customized intra-oral mould technique. Radiat Med 2007; 25: 181-186.

12. Kudoh T, Ikushima H, Kudoh KT et al. High-dose-rate brachytherapy for patients with maxillary gingival carcinoma using a novel customized intraoral mould technique. Oral Surg Oral Med Oral Pathol Oral Radiol Endod 2010; 109: e102-108.

13. Chatani M, Tsuboi K, Yagi M et al. High dose rate brachytherapy using moulds after chemoradiotherapy for oral cavity cancer. Jpn J Radiol 2012; 30: 40-44.

14. Matsuzaki H, Takemoto M, Hara M et al. Two-piece customized mould technique for high-dose-rate brachytherapy on cancers of the buccal mucosa and lip. Oral Surg Oral Med Oral Pathol Oral Radiol Endod 2012; 113: 118-125.

15. Nag S. High dose rate brachytherapy: its clinical applications and treatment guidelines. Technol Cancer Res Treat 2004; 3: 269-287. 